\title{
Index One Minimal Surfaces in Flat Three Space Forms
}

\author{
MANUel RitorÉ
}

\begin{abstract}
In this paper we are concerned with complete orientable index one minimal surfaces properly embedded in orientable flat three space forms. We give an almost complete classification of index one noncompact surfaces which provides a bound on their total curvature. Using this bound we study the spaces of compact index one minimal surfaces in flat three space forms. We also give an application of our results to the study of volume preserving stable surfaces in three manifolds with non-negative Ricci curvature.
\end{abstract}

\section{INTRODUCTION}

It is known that minimal surfaces are the critical points for the area functional in Riemannian manifolds. If we require more information about these critical points we are led to consider the second variation of the area. In order to compute it, let $\psi: M \rightarrow N$ be a minimal immersion from a complete orientable surface into an orientable Riemannian three manifold and let $\nu$ be a smooth choice of unit normal. If $\psi_{t}$ is a variation of $\psi$ with initial velocity vector field $V=\left(d \psi_{t} / d t\right)_{t=0}$, and $u=\langle V, \nu\rangle$ is its normal component, then the second derivative of the area is given by

$$
I(u)=\left.\frac{d^{2}}{d t^{2}}\right|_{t=0} \operatorname{area}\left(\psi_{t}(M)\right)=\int_{M}\left\{|\nabla u|^{2}-\left(\operatorname{Ric}(\nu)+|\sigma|^{2}\right) u^{2}\right\} d A,
$$

where Ric is the Ricci curvature of the manifold $N$ and $|\sigma|^{2}$ is the square of the norm of the second fundamental form $\sigma$ of $\psi$ (the sum of the squared principal curvatures of $\psi$ ). The expression $I(u)$, called the index form of $M$, is the quadratic form associated to the Jacobi operator $\Delta+\operatorname{Ric}(\nu)+|\sigma|^{2}$, where $\Delta$ is the Laplacian on $M$. 
For any bounded domain $\Omega \subset M$ the index of $\Omega$ is the number of negative eigenvalues of $L$ on $\Omega$. The index of $M$ is the supremum of the indexes of $\Omega$, when $\Omega$ varies over all bounded domains in $M$.

Index zero minimal surfaces are second order minima of the area for any variation with compact support and are usually called stable minimal surfaces. This kind of surfaces has been studied by Fischer-Colbrie and Schoen [FCS] when $M$ is complete and orientable and $N$ is a complete orientable three manifold with non-negative scalar curvature. If the Ricci curvature of $N$ is non-negative, it follows from Fischer-Colbrie results $[\mathrm{FC}]$ that a stable orientable minimal surface must be totally geodesic. See also $[\mathrm{CP}]$ for an earlier proof when $N=\mathbb{R}^{3}$.

From a variational point of view, the "simplest" non totally geodesic minimal surfaces are index one minimal surfaces. These surfaces were constructed by Pitts $[\mathrm{P}]$ on a wide class of Riemannian manifolds by means of a minimax method. They have been used to obtain interesting topological properties of the ambient manifolds. We should cite that Frohman and Hass $[\mathrm{FH}]$, using the minimax construction and certain properties of minimal surfaces, proved the uniqueness of genus three Heegaard splittings in a three torus. Later, Pitts and Rubinstein $[\mathrm{PR}]$ obtained minimal surfaces in geometric three manifolds and Hass, Pitts and Rubinstein [HPR] constructed minimal surfaces in flat three tori. It is worth noting that the minimax construction yields both orientable and nonorientable surfaces.

The aim of this paper is to study complete orientable index one minimal surfaces embedded in orientable flat three space forms. Our first result, Proposition 1.1, allows to control the topological type of an index one minimal surface in a 3-manifold with non-negative Ricci curvature by using holomorphic maps from the surface to $\mathbb{S}^{2}$ with bounded energy, $[\mathrm{Y}]$. Combining this result with another one about Schrödinger operators associated to a holomorphic map, Proposition 1.2, we prove in Theorem 1.3:

A complete orientable index one minimal surface immersed in a complete orientable three manifold with non-negative Ricci curvature is conformally equivalent to a compact Riemann surface of genus $g \leq 4$ minus $r \leq 4-g$ points.

In Section 2 we study noncompact index one minimal surfaces in orientable flat three space forms. We prove in Theorem 2.1 the following result:

A noncompact orientable index one minimal surface properly embedded in an orientable flat three space form form must be one of the following:

(i) a catenoid in $\mathbb{R}^{3}$,

(ii) a quotient of a singly-periodic Scherk surface by a translation which has genus zero and four ends,

(iii) a quotient of a doubly periodic Scherk surface by a commensurable lattice which has genus zero and four ends, 
(iv) a quotient of a helicoid by a screw motion which has total curvature greater than or equal to $-4 \pi$, or

(v) a two ended torus with total curvature strictly greater than $-8 \pi$ in a quotient of $\mathbb{R}^{3}$ by a discrete subgroup of isometries which contains not only translations.

We will show in Section 2 that certain quotients of the singly-periodic Scherk surfaces yield index one minimal tori with two Scherk type ends which are properly embedded in the quotient of $\mathbb{R}^{3}$ by a screw motion, giving examples of the last possibility in the theorem above. For quotients of $\mathbb{R}^{3}$ by discrete subgroups of translations we obtain Proposition 3.1 in [RR2].

A consequence of the result above is a bound on the total curvature $C(M)$ of a complete noncompact index one minimal surface $M$. We obtain for such surfaces, see Corollary 2.2, that

$$
C(M)=\int_{M} K>-8 \pi
$$

where $K$ is the Gauss curvature of the surface. We use this result in Section 3, where we study the spaces of compact index one minimal surfaces in flat three space forms. In Theorem 3.1 we prove the following result:

The space of index one minimal surfaces with genus greater than two in flat three space forms is compact.

This compactness will always be understood in a strong sense: we have convergence of a subsequence of both the surfaces and the ambient manifolds. More precisely: suppose that we have a sequence of properly embedded minimal surfaces $M_{n} \subset \mathbb{R}^{3}$ which are invariant by discrete subgroups of isometries $G_{n}$ and such that $M_{n} / G_{n}$ is an embedded orientable index one minimal surface in the manifold $\mathbb{R}^{3} / G_{n}$, which has injectivity radius one. Then there exist subsequences $M_{j}, G_{j}$ such that:

(i) $M_{j}$ converges in the $C^{k}$-topology, $k \geq 2$, to a properly embedded minimal surface $M$ in $\mathbb{R}^{3}$ with multiplicity one,

(ii) $G_{j}$ converges in the isometries group to a group $G$, and

(iii) $M$ is invariant by $G$ and $M / G$ is a compact index one minimal surface embedded in $\mathbb{R}^{3} / G$, which is diffeomorphic to $\mathbb{R}^{3} / G_{j}$ for $j$ large enough.

This result was proved for index one minimal surfaces in flat three tori in [RR2]. The lower bound on the genus is necessary since there exists a noncompact family of compact index one minimal surfaces with genus two.

Finally we apply our results in Section 4 to show in Theorem 4.1 that the genus of a compact volume preserving stable surface in a three manifold with non-negative Ricci curvature must be less than or equal to four. This improves 
slightly the best known bound on the genus, see [RR1, Theorem 2] and the references there.

This work originated from the author's Ph.D. Thesis. I want to express particular thanks to my advisor, Prof. Antonio Ros, for his kind help and advice during the preparation of this paper. I would also like to thank Prof. Gian Pietro Pirola for many valuable conversations about algebraic curves.

\section{BASIC RESUlts}

We start this section with the following restriction on the topological type of an index one minimal surface

Proposition 1.1. Let $M$ be a complete orientable index one minimal surface immersed in a complete orientable three manifold with non-negative Ricci curvature. Then $M$ is conformally equivalent to a compact Riemann surface of genus $g \leq 5$ minus $r$ points and

$$
r \leq 4+2\left[\frac{1+g}{2}\right]-2 g
$$

where $[x]$ is the largest integer less than or equal to $x$.

Moreover, if equality holds in (1) then there exists a \pm holomorphic map $\phi: \bar{M} \rightarrow \mathbb{S}^{2}$ of degree $1+[(1+g) / 2]$ such that $|\nabla \phi|^{2}=\operatorname{Ric}(\nu)+|\sigma|^{2}=-2 K$.

Proof. Since $M$ has finite index it is conformally a compact Riemann surface $\bar{M}$ of genus $g$ minus $r$ points by the work of Fischer-Colbrie [FC, Theorem 1]. Let $d s^{2}$ be the Riemannian metric on $M, \Delta$ the Laplacian for the metric $d s^{2}$ and let $q=\operatorname{Ric}(\nu)+|\sigma|^{2}$. With this notation the Jacobi operator is $\Delta+q$.

Consider on $M$ a smooth function $\rho>0$ so that the metric $h=\rho d s^{2}$, which is conformal to $d s^{2}$, be complete and with finite area. Let $\Delta_{h}$ be the Laplacian on $(M, h)$. The operators $\Delta+q$ and $\Delta_{h}+\rho^{-1} q$ have the same index since the associated quadratic forms coincide because of the conformal invariance of the Dirichlet integral $\int_{M}|\nabla u|^{2}$.

As $M$ has index one, Proposition 2 in [FC] implies the existence of an eigenfunction $f_{1} \in L_{2}(M, h)$ for the operator $\Delta_{h}+\rho^{-1} q$ whose associated eigenvalue is negative. Such a function is globally defined on $M$ and we have $I(u) \geq 0$ for all $u \in C_{0}^{\infty}(M)$ which are $L_{2}(M, h)$-orthogonal to $f_{1}$. By construction (see the proof of Proposition 2 in [FC]), the function $f_{1}$ is non negative. Since it is smooth by elliptic regularity it is strictly positive by the maximum principle.

Claim. Inequality $I(u) \geq 0$ holds for any $u \in C^{\infty}(\bar{M})$ which is $L_{2}(M, h)$ orthogonal to $f_{1}$.

Note that $I(u)$ is finite since $q \in L_{1}\left(M, d s^{2}\right)$ by [FC, Corollary 1], and that $u f_{1}$ is an integrable function on $(M, h)$ by the Schwarz inequality. In order to 
prove the claim we consider conformal coordinates $z_{i}$ centered at the points in $\bar{M}-M$ and define, for $\varepsilon>0$ small enough, the functions

$$
v_{\varepsilon}\left(z_{i}\right)= \begin{cases}0, & \left|z_{i}\right|<\varepsilon^{2}, \\ \frac{\log \left(|z| / \varepsilon^{2}\right)}{\log (1 / \varepsilon)}, & \varepsilon^{2} \leq|z| \leq \varepsilon, \\ 1, & \left|z_{i}\right|>\varepsilon,\end{cases}
$$

extended by 1 to the entire surface. It follows easily that $v_{\varepsilon} \geq 0$, that $v_{\varepsilon} \nearrow 1$ pointwise when $\varepsilon \rightarrow 0$ and that the Dirichlet integral of $v_{\varepsilon}$ tends to 0 when $\varepsilon \rightarrow 0$.

Now consider $u \in C^{\infty}(\bar{M})$ orthogonal to $f_{1}$ in $L_{2}(M, h)$. Choose $\mu_{\varepsilon}$ so that the function $u_{\varepsilon}=\mu_{\varepsilon}\left(v_{\varepsilon} u\right)^{+}-\left(v_{\varepsilon} u\right)^{-}$is $L_{2}(M, h)$-orthogonal to $f_{1}$. Then $\mu_{\varepsilon} \rightarrow 1$ when $\varepsilon \rightarrow 0$. As $u_{\varepsilon}$ has compact support on $M$ and it is $L_{2}(M, h)$-orthogonal to $f_{1}$ we have $I\left(u_{\varepsilon}\right) \geq 0$. Letting $\varepsilon \rightarrow 0$, we see that $I\left(u_{\varepsilon}\right) \rightarrow I(u) \geq 0$ and the claim follows. See [FC, Theorem 2] for a similar computation.

Let $\phi: \bar{M} \rightarrow \mathbb{S}^{2}$ be a holomorphic map with degree less than or equal to $1+[(1+g) / 2],[\mathrm{GH}]$. As $(M, h)$ has finite area, we can suppose, after composing $\phi$ with a Möbius transformation of $\mathbb{S}^{2}$, that the coordinates of $\phi$ are $L_{2}(M, h)$ orthogonal to $f_{1}$, [LY]. By the claim above we conclude $\sum_{i=1}^{3} I\left(\phi_{i}\right) \geq 0$. Writing the index form in terms of the metric $d s^{2}$, we have

$$
\begin{aligned}
8 \pi \operatorname{deg} \phi & =\int_{M}|\nabla \phi|^{2} d A=\sum_{i=1}^{3} \int_{M}\left|\nabla \phi_{i}\right|^{2} d A \\
& \geq \int_{M}\left(\operatorname{Ric}(\nu)+|\sigma|^{2}\right) d A
\end{aligned}
$$

where $d A$ is the area element in $\left(M, d s^{2}\right)$. Taking into account that $M$ is minimal and that the Ricci curvature of $N$ is non-negative, we deduce that

$$
\operatorname{Ric}(\nu)+|\sigma|^{2}=\operatorname{Ric}\left(e_{1}\right)+\operatorname{Ric}\left(e_{2}\right)-2 K \geq-2 K,
$$

where $K$ is the Gauss curvature of $\left(M, d s^{2}\right)$ and $\left\{e_{1}, e_{2}\right\}$ is an orthonormal basis tangent to $M$. Using (3) together with Cohn-Vossen inequality $\int_{M} K \leq$ $2 \pi(2-2 g-r)$, we see from (2) that inequality (1) holds.

Suppose now that equality holds in (1). Then we also get equality in (3) and so

$$
-2 K=\operatorname{Ric}(\nu)+|\sigma|^{2} .
$$

Equality in (2) also holds and so $I\left(\phi_{i}\right)=0$ for all $i$. Consider a smooth function $u$ on $M$ with compact support and $L_{2}(M, h)$-orthogonal to $f_{1}$. Then $I\left(\phi_{i}+t u\right) \geq 0$ 
for real $t$ because of the claim above and so $(d / d t)_{t=0} I\left(\phi_{i}+t v\right)=0$ for all $i$. Since

$$
\left.\frac{d}{d t}\right|_{t=0} I\left(\phi_{i}+t v\right)=\int_{M}\left\{h\left(\nabla_{h} \phi_{i}, \nabla_{h} v\right)-\rho^{-1} q \phi_{i} v\right\} d A_{h},
$$

where $\nabla_{h}$ and $d A_{h}$ are the gradient and the area element on $(M, h)$, respectively, it follows that $\Delta_{h} \phi+\rho^{-1} q \phi=c f_{1}$ for some vector $c \in \mathbb{R}^{3}$, and we conclude from (4) that $\Delta \phi-2 K \phi=c \rho f_{1}$. On the other hand, since $\phi: M \rightarrow \mathbb{S}^{2}$ is a harmonic map we have $\Delta \phi+|\nabla \phi|^{2} \phi=0$. From both equations we obtain that $\left(|\nabla \phi|^{2}+2 K\right) \phi=c \rho f_{1}$ and, since $\phi$ is non constant, we deduce that $c=0$ and that $|\nabla \phi|^{2}=-2 K$.

When $r=4+2[(1+g) / 2]-2 g$, Proposition 1.1 shows that $\operatorname{Ric}(\nu)+|\sigma|^{2}=$ $|\nabla \phi|^{2}$ for some \pm holomorphic map $\phi: M \rightarrow \mathbb{S}^{2}$. The operators of the type $\Delta+|\nabla \phi|^{2}$, where $\phi: M \rightarrow \mathbb{S}^{2}$ is a \pm holomorphic map, have been studied by Montiel and Ros, $[\mathrm{MoR}]$. They are often referred to as operators associated to the holomorphic map $\phi$. The following result is based on $[\mathrm{MoR}]$ :

Proposition 1.2. Let $M$ be a compact Riemann surface, and $L=\Delta+$ $|\nabla \phi|^{2}$ the Schrödinger operator associated to the \pm holomorphic map $\phi: M \rightarrow$ $\mathbb{S}^{2}$. Then:

(i) If genus $M=1$, then index $L>1$.

(ii) If genus $M=3$ and index $L=1$, then $\operatorname{deg} \phi=2$ and so $M$ is a hyperelliptic surface.

(iii) If genus $M=5$ and $\operatorname{deg} \phi=4$, then index $L>1$.

Proof. Statement (i) was proved in [MoR, Corollary 8]. We shall show (ii) and (iii) by using Theorem 6 in $[\mathrm{MoR}]$ :

Suppose that $\phi: M \rightarrow \mathbb{S}^{2}$ is a holomorphic map on a Riemann surface and that the index of the operator $\Delta+|\nabla \phi|^{2}$ is one. Then there are no holomorphic maps on $M$ with degree less than $\operatorname{deg} \phi$. Moreover, if $\eta: M \rightarrow \mathbb{S}^{2}$ is a holomorphic map with $\operatorname{deg} \phi=$ $\operatorname{deg} \eta$ then $\phi$ and $\eta$ are related by a Möbius transformation, i.e., there exists a conformal transformation $A$ of $\mathbb{S}^{2}$ such that $\phi=$ $A \circ \eta$.

Parts (ii) and (iii) can be proved by using Brill-Noether theory. We shall give elementary proofs.

Let us see (ii). Suppose that $M$ is non hyperelliptic. We shall prove the existence of meromorphic maps which are not related by a Möbius transformation. Choose a basis of abelian differentials and consider the associated canonical curve $f: M \rightarrow \mathbb{C P}^{2}$, which is an embedding since $M$ is not hyperelliptic. The 
degree $d$ of $f(M)$ equals $2 g-2=4$. Fix a projective line $L \subset \mathbb{C P}^{2}$ meeting $f(M)$ at $d$ different points (a generic line verifies this property). Projecting from a point in $f(M)-L$ to $L$, we obtain a meromorphic map with degree $d-1=3$. We claim that projecting from different points in $f(M)-L$, we obtain meromorphic maps which are not related by a Möbius transformation: indeed, if they were related then there would exist a projective transformation $A$ of $L$ relating both projections; but $A$ would fix the points in $f(M) \cap L$ and it would be the identity map on $L$, so implying that we are projecting from the same point. This contradiction proves the claim and finishes the proof of (ii).

Let us see now (iii). The result by Montiel and Ros implies that $M$ is neither hyperelliptic nor trigonal (no holomorphic $3: 1$ map to $\mathbb{S}^{2}$ ). Fix two different points $p$ and $q$ on $M$. The Riemann-Roch Theorem implies that the space $V$ of abelian differentials vanishing at $p$ and $q$ has dimension three since $M$ is not hyperelliptic. Another application of the Riemann-Roch Theorem in which $M$ is not trigonal shows that, for any $x \neq p, q$, there exists an element of $V$ not vanishing at $x$. Thus, choosing a basis of $V$, one can define a map from $M-\{p, q\}$ to $\mathbb{C P}^{2}$ that can be easily extended to a map $f: M \rightarrow \mathbb{C P}^{2}$. A third application of the Riemann-Roch Theorem shows that there are one forms in $V$ vanishing either at $p$ or $q$ with order one. So if $g=$ genus $M$, then the degree $d$ of $f(M)$ equals $2 g-2-\operatorname{deg}(p+q)=6$. The curve $f(M)$ must be singular since otherwise the genus formula would imply that $g$ equals $(d-1)(d-2) / 2=10$. Projecting from a singular point with multiplicity $m$ to a line, we get a meromorphic map with degree $d-m=6-m$. As $6-m \geq 4$, by the Montiel and Ros result cited above we obtain $m=2$. The genus formula then implies that there are 5 singular points. An easy argument shows that no more than two singular points can be applied onto the same point by $f$ : it can be supposed that such a point is $P=[(0,0,1)]$. If there are three singular points mapped onto $P$ and $f=\left[\left(\omega_{0}, \omega_{1}, \omega_{2}\right)\right]$, then $\omega_{0}$ and $\omega_{1}$ are linearly independent and they have the same divisors of zeroes, giving us a contradiction. So projecting from different singular points to a line meeting $f(M)$ at 6 different points, we obtain at least three meromorphic maps which are not related by a Möbius transformation.

From Propositions 1.1 and 1.2 we obtain the following result:

Theorem 1.3. Let $M$ be a complete orientable index one minimal surface immersed in a complete orientable three manifold with non-negative Ricci curvature. Then $M$ is conformally a compact Riemann surface of genus $g \leq 4$ minus $r \leq 4-g$ points.

To end this section we state a restriction on the type of a flat three space form containing a minimal surface with a \pm holomorphic map $\phi$ satisfying $|\nabla \phi|^{2}=$ $-2 K$. 
Proposition 1.4. Let $M$ be an orientable minimal surface properly embedded in an orientable flat three space form $N$. Suppose that there exists on $M$ a non constant \pm holomorphic map $\phi: M \rightarrow \mathbb{S}^{2}$ such that $|\nabla \phi|^{2}=-2 K$.

Then $N$ is the quotient of $\mathbb{R}^{3}$ by a discrete subgroup of translations and $\phi$ is, up to rigid motions, the Gauss map of $M$.

Proof. As $-2 K=|\nabla \phi|^{2} \not \equiv 0$, it follows that $M$ is not flat. Let $M^{\prime}$ be the pullback of $M$ via the covering map $\mathbb{R}^{3} \rightarrow N$. The surface $M^{\prime}$ is connected by the halfspace theorem [HM1]. Let $\nu$ be the Gauss map of $M^{\prime}$ and let $\phi^{\prime}$ be the lifting of $\phi$ to $M^{\prime}$.

From equality $\left|\nabla \phi^{\prime}\right|^{2}=-2 K^{\prime}$, where $K^{\prime}$ is the Gauss curvature of $M^{\prime}$, we conclude $|\nabla \nu|^{2}=\left|\nabla \phi^{\prime}\right|^{2}$. As $M^{\prime}$ is connected, an argument by M. Ross [Ro2, p. 68] shows that there exists an isometry $A$ of $\mathbb{S}^{2}$ such that $\nu=A \circ \phi^{\prime}$.

Consider a transformation of the covering group $f=t \circ u$, where $t$ is a translation and $u$ is a linear isometry. We shall prove that $u$ is the identity map, which clearly implies the result. Choose $p \in M^{\prime}$. Since $M^{\prime}$ is invariant by $f$ and $\phi^{\prime}$ is a lifting of $\phi$ we see that $\phi^{\prime}(p)=\phi^{\prime}(f(p))$. Composing with $A$, we obtain $\nu(p)=\nu(f(p))$. Since the normal $\nu$ is equivariant with respect to the covering group it follows that $\nu(f(p))=u(\nu(p))$. We conclude $u(\nu(p))=\nu(p)$ for all $p \in M^{\prime}$. As $M$ is not totally geodesic, it follows that the map $\nu$ is open and we deduce from the last equality that $u$ is the identity.

An important fact to be used later, which is proved from Theorem 1.3 and from Proposition 1.4, is as follows:

Proposition 1.5. Let $M \subset N$ be an orientable index one minimal surface properly embedded in an orientable flat three space form $N$.

Then for any holomorphic map $\phi: M \rightarrow \mathbb{S}^{2}$ the total curvature $C(M)$ of $M$ satisfies $|C(M)| \leq 4 \pi \operatorname{deg} \phi$. Moreover, if $|C(M)|=4 \pi \operatorname{deg} \phi$, then the Jacobi operator is given by $\Delta+|\nabla \phi|^{2}$ and $N$ is the quotient of $\mathbb{R}^{3}$ by a discrete subgroup of translations.

Proof. Trace the proof of Proposition 1.1 to obtain inequality (2), which implies $|C(M)| \leq 4 \pi \operatorname{deg} \phi$ in a flat ambient manifold. If equality holds, then $I\left(\phi_{i}\right)=0$ for all the coordinates $\phi_{i}$ of the map $\phi$. The last part of the proof of Proposition 1.1 then shows that $|\nabla \phi|^{2}=-2 K$ and we conclude by applying Proposition 1.4.

\section{NONCOMPACT INDEX ONE MiNimal SURFACES}

There are many known examples of properly embedded index one minimal surfaces in quotients of $\mathbb{R}^{3}$. López and Ros $[\mathrm{LR}]$ proved that the catenoid and Enneper surface are the only complete orientable index one minimal surfaces in $\mathbb{R}^{3}$, and so the catenoid is the only properly embedded one. The singly and 
doubly periodic Scherk surfaces with genus zero and four ends have Gauss maps of degree one and, from [MoR, Corollary 15] their index is one. M. Soret [S] have computed the index of any quotient of the helicoid by a screw motion leaving invariant the surface. It follows that helicoids obtained in such a way with total curvature greater than or equal to $-4 \pi$ have index one.

We are going now to construct an example of an index one minimal surface in a flat three space form with genus one and two ends. Consider the quotient $M$ of the singly-periodic Scherk surface whose conformal structure and Weierstrass representation are given by

$$
\begin{gathered}
w^{2}=\left(z-e^{i \theta}\right)\left(z+e^{i \theta}\right)\left(z-e^{-i \theta}\right)\left(z+e^{-i \theta}\right)-\left\{\left( \pm e^{ \pm i \theta}, 0\right)\right\} \\
g(z, w)=z, \quad \omega(z, w)=\frac{d z}{w^{2}}
\end{gathered}
$$

The surface $M$ is a minimal torus with four Scherk type ends which is properly embedded in a quotient of $\mathbb{R}^{3}$ by a translation. The surface $M$ is invariant by a screw motion $S$ consisting on a rotation $R$ of angle $\pi$ and a translation $T$ in the direction of the axis of the screw motion. The restrictions of the above isometries to $M$ are given, respectively, by $\sigma(z, w)=(-z,-w), \sigma_{1}(z, w)=(-z, w)$ and $\theta(z, w)=(z,-w)$. The anticonformal involutions $h_{1}(z, w)=(-\bar{z}, \bar{w})$ and $h_{2}(z, w)=(\bar{z}, \bar{w})$ are intrinsic isometries of $M$ that preserve the Gauss curvature and extend to symmetries with respect to planes parallel to $x_{1}=0$ and $x_{2}=0$. The surface $M / S$ is an embedded minimal surface in $\mathbb{R}^{3} / S$ with genus one and two Scherk type ends.

Let $\phi: M \rightarrow \mathbb{S}^{2}$ be the Gauss map of $M$. The spherical metric $d s_{\phi}^{2}=\phi^{*} d s_{0}^{2}$, where $d s_{0}^{2}$ is the standard metric of $\mathbb{S}^{2}$, is invariant by $S$ and hence it is induced on $M / S$. This metric extends smoothly to the ends of $M$. So we can compute the index of $M / S$ as the number of negative eigenvalues of $\Delta_{\phi}+2$ on $M$ whose associated eigenfunctions are invariant by $\sigma\left(\left[\mathrm{FC}\right.\right.$, Theorem 2]). Here $\Delta_{\phi}$ is the Laplacian of the branched metric $d s_{\phi}^{2}([\mathrm{MoR}])$. It is worth noting that the index of $M$ is three since the branching values of the Gauss map lie on an equator of $\mathbb{S}^{2}$ ([MoR, Corollary 15]) although this information will not be used in our reasonings.

Let $u$ be a non constant eigenfunction of $\Delta_{\phi}+2$ with negative eigenvalue. Let us see that $u$ is antisymmetric with respect to $\theta$. Since $\theta$ is an involution $u$ can be decomposed as the sum of a symmetric $u_{e}=(u+u \circ \theta) / 2$ eigenfunction and an antisymmetric $u_{o}=(u-u \circ \theta) / 2$ one. The function $u_{e}$ can be projected to $\mathbb{S}^{2}$ and, if it were non constant it would provide a non constant eigenfunction for the Laplacian of $\mathbb{S}^{2}$ with eigenvalue strictly less than 2 . This contradiction shows that $u_{e} \equiv 0$ and so $u \circ \theta=-u$ ([So, p. 374] $)$.

On the other hand, $u$ is symmetric with respect to $h_{1}$ and $h_{2}$. Let us check it for $h_{1}$. The set of fixed points of $h_{1}$ divides $M$ in two connected components $A$ and $B$. The coordinate $\phi_{1}$ of the Gauss map is a positive eigenfunction 
with eigenvalue 0 for the Dirichlet problem on $A$ and $B$, and so it is the first eigenfunction. As before we can write $u=u_{e}+u_{o}$, where $u_{e}$ is symmetric and $u_{o}$ antisymmetric with respect to $h_{1}$. If $u_{o}$ were non zero then its restriction to $A$ and $B$ would be an eigenfunction with negative eigenvalue for the Dirichlet problem on $A$ and $B$, which is not possible since the first eigenvalue is 0 . Hence $u=u_{e}$ and so $u$ is symmetric with respect to $h_{1}$. The proof for $h_{2}$ is similar.

As $\sigma=\left(h_{1} \circ h_{2}\right) \circ \theta$ we deduce that the non constant eigenfunctions with negative eigenvalue of $\Delta_{\phi}+2$ are antisymmetric with respect to $\sigma$ and so the index of $M / S$ is one.

Let us prove now that there are no quotients of the doubly-periodic Scherk surface with genus one and two ends which have index one. Consider now the quotient $M$ of the doubly-periodic Scherk surface whose Weierstrass representation is given by

$$
\begin{gathered}
w^{2}=\left(z-e^{i \theta}\right)\left(z+e^{i \theta}\right)\left(z-e^{-i \theta}\right)\left(z+e^{-i \theta}\right)-\left\{\left( \pm e^{ \pm i \theta}, 0\right)\right\} \\
g(z, w)=z, \quad \omega(z, w)=\frac{\mathrm{i} d z}{w^{2}}
\end{gathered}
$$

The surface $M$ is a genus one minimal surface with four Scherk type ends which is properly embedded in the quotient of $\mathbb{R}^{3}$ by a rank-two discrete subgroup of translations. Note that $M$ is invariant by certain screw motions consisting on rotations of angle $\pi$ followed by a translation in the direction of the axis of the screw motion, which is contained in the $x_{1} x_{2}$-plane. The third coordinate of the Gauss map is antisymmetric with respect to any screw motion $S$ leaving invariant the surface and axis contained in the $x_{1} x_{2}$-plane. Let us see that the surface $M / S$ cannot have index one.

The nodal set of $\phi_{3}$ on $M$ consists on four components $\Omega_{i}, i=1, \ldots, 4$, which we can label in such a way that $S\left(\Omega_{1}\right)=\Omega_{2}$ and $S\left(\Omega_{3}\right)=\Omega_{4}$. Call $u_{1}$ and $u_{3}$ to the restrictions of $\phi_{3}$ to $\Omega_{1}$ and $\Omega_{3}$, respectively.

If the index of $M / S$ were one then the second eigenvalue of the operator $\Delta_{\phi}+2$ on $M / S$ ( $d s_{\phi}^{2}$ can be induced on $M / S$ and extends smoothly to the ends) would be greater than or equal to zero. The functions $u_{1}$ and $u_{3}$ can be projected to $M / S$ and, since their supports are disjoint, a linear combination $u$ of both is orthogonal to the first eigenfunction. This implies that $u$ is an eigenfunction of $\Delta_{\phi}+2$ with eigenvalue 0 . Hence $u$ is smooth and its lifting $v$ to $M$ is symmetric with respect to $\sigma$. But $v$ is proportional to $\phi_{3}$ on $\Omega_{1}$ and, by unique continuation, on $M$. This gives us a contradiction since $v$ is symmetric and $\phi_{3}$ is antisymmetric with respect to $S$.

The helicoidal saddle towers discovered by Karcher [Kar1] are invariant by screw motions so that certain quotients are minimal tori with two helicoid type ends. We do not know if their index is one.

For noncompact surfaces we obtain the following result. 
Theorem 2.1. Let $M$ be a noncompact orientable index one minimal surface properly embedded in an orientable flat three space form $N$. Then only one of the following possibilities holds

(i) $M$ is a catenoid in $\mathbb{R}^{3}$,

(ii) $M$ is the quotient of a singly-periodic Scherk surface by a translation and it has genus zero, four ends, and total curvature $-4 \pi$,

(iii) $M$ is the quotient of a doubly periodic Scherk surface by a commensurable lattice and it has genus zero, four ends, and total curvature $-4 \pi$,

(iv) $M$ is the quotient of a helicoid by a screw motion and it has genus zero, two ends and total curvature greater than or equal to $-4 \pi$.

(v) $M$ is a two ended torus with total curvature strictly greater than $-8 \pi$ in a quotient of $\mathbb{R}^{3}$ by a subgroup of isometries which contains not only translations.

Remark. All the surfaces in items (i)-(iv) in the statement of Theorem 2.1 have index one. This has been showed in the discussion at the beginning of this section. We have also proved that the class of minimal surfaces described in (v) have examples of index one minimal surfaces, and that not all the surfaces in this class have index one.

The reader can consult [Wo, p. 112] for a list of affine diffeomorphism classes of complete noncompact flat three manifolds.

Proof. Suppose that $M$ is conformally equivalent to a compact Riemann surface minus $r$ points, with $r \neq 0$. The surface $M$ cannot be totally geodesic since its index is not zero.

If $N=\mathbb{R}^{3}$, then $M$ must be a catenoid by the work by López and Ros [LR]. If $N$ is not simply connected then $M$ must have an even number of ends [MR2, Theorem 8]. So from Theorem 1.3 the only possibilities we must consider are

$$
\begin{array}{ll}
g=0 & r=2,4, \\
g=1 & r=2, \\
g=2 & r=2,
\end{array}
$$

If $g=2$, then we get equality in (1) and so the Jacobi operator is given by the Schrödinger operator associated to a holomorphic map $\phi$ of degree 2 such that $|\nabla \phi|^{2}=-2 K$. By Proposition 1.4 the ambient manifold $N$ is the quotient of $\mathbb{R}^{3}$ by a discrete subgroup of translations of rank one or two. The latter case is not possible because an orientable properly embedded minimal surface in the product of a flat two torus with the real line has at least four ends by [MR1]. Hence $N$ is the quotient of $\mathbb{R}^{3}$ by the subgroup generated by a translation. The total curvature $C(M)$ of $M$ equals $-4 \pi \operatorname{deg} \phi=-8 \pi$, which coincides with $2 \pi \chi(M)$, and implies that the two ends of $M$ are of Scherk type. This is not possible since in this case we could lift $M$ to $\mathbb{R}^{3}$ as a properly embedded minimal 
surface contained in a halfspace, contradicting the results by Hoffman and Meeks [HM2].

Suppose now that $g=1$ and $r=2$. As we can find on $M$ a holomorphic map $\phi$ of degree 2, Proposition 1.5 shows that $C(M) \geq-8 \pi$. If $C(M)=-8 \pi$, then Proposition 1.5 implies that the Jacobi operator on $M$ is the one associated to a holomorphic map, which yields a contradiction by (i) in Proposition 1.2.

If $g=0$ and $r=4$, then it follows from Propositions 1.1 and 1.4 that $N$ is the quotient of $\mathbb{R}^{3}$ by a discrete subgroup of translations. We deduce from [HM1] and [MR1] that $M$ must be a singly or doubly periodic Scherk surface with genus zero, four ends and total curvature $-4 \pi$.

To end the proof consider the case $g=0$ and $r=2$. The manifold $N$ cannot be a product $\mathbb{T} \times \mathbb{R}$, where $\mathbb{T}$ is a flat two torus, since orientable properly embedded minimal surfaces in such manifolds have at least four ends.

Following with the case $g=0, r=2$, we suppose now that $N$ is of type $\mathcal{K}_{1}$, [Wo, p. 112]. The manifold $N$ can be seen as the quotient of $\mathbb{T} \times \mathbb{R}$ by a screw motion $S$ well defined on $\mathbb{T} \times \mathbb{R}$ whose axis is contained in the $x_{1} x_{2}$ plane and it is involutive in $\mathbb{T} \times \mathbb{R}$. Let us see that a two ended plane properly embedded as a minimal surface in $N$ is totally geodesic and hence it has index zero. The lifting $M^{\prime}$ of $M$ to $\mathbb{T} \times \mathbb{R}$ is a properly embedded orientable minimal surface and so it has four ends [MR1]. The top and the bottom ends of $M^{\prime}$ are permuted by $S$ and we deduce that each end of $M$ is congruent to an end of $M^{\prime}$, which are of Scherk type. The total curvature of a minimal surface with Scherk type ends can be computed from the Gauss-Bonnet formula as in the proof of Theorem 1 in [MR1, pp. 358-359] and it is equal to the Euler characteristic $\chi(M)$. In our particular case $\chi(M)=0$ and so the surface is totally geodesic and hence it has index zero, which gives us a contradiction since $M$ is assumed to have index one.

To end the case $g=0, r=2$, suppose that $M$ is embedded in a quotient of $\mathbb{R}^{3}$ by a helicoidal motion (which could be a translation). The surface $M$ is a minimally embedded annulus with finite, non zero total curvature, since it has index one and it is not stable. So $M$ satisfies the hypotheses of Theorem 8 in [MR2, p. 547] and it must be a helicoid. Using a degree one holomorphic map, we see from Proposition 1.5 that $C(M) \geq-4 \pi$.

Remark. The ambient manifolds for the surfaces in (v) can be of two types:

(i) manifolds of type $\mathcal{K}_{1}$ ([Wo, p. 112]), where index one minimal surfaces would have total curvature $-4 \pi$. We have not been able to produce an example of an index one minimal surface in such manifolds;

(ii) the quotient of $\mathbb{R}^{3}$ by a screw motion with non trivial rotational part. Minimal surfaces in such quotients have ends of helicoid, planar or Scherk type. 
Examples of index one tori with two Scherk type ends were presented at the beginning of the section, and they all have total curvature $C(M)=-4 \pi$. There exist also examples of properly embedded minimal tori with two ends of helicoid type and they have total curvature smaller than $-4 \pi$ ([MR2]). Hence the total curvature of an index one minimal torus with two helicoidal ends lies in the interval $(-8 \pi,-4 \pi)$. Minimal tori with two embedded planar ends have total curvature $-8 \pi$ and hence they cannot have index one by Theorem 2.1.

An easy consequence of Theorem 2.1 is the following bound on the total curvature for an orientable index one minimal surface

Corollary 2.2. Let $M$ be a complete orientable noncompact index one minimal surface properly embedded in an orientable flat three manifold $N$. Then $C(M)>-8 \pi$.

Theorem 2.1 implies the following result when the ambient manifolds is a quotient of $\mathbb{R}^{3}$ by translations ([RR2, Proposition 3.1]).

Corollary 2.3. Let $M$ be a complete orientable noncompact index one minimal surface properly embedded in a quotient of $\mathbb{R}^{3}$ by a discrete subgroup of translations. Then $M$ is either

(i) a catenoid in $\mathbb{R}^{3}$, or

(ii) $M$ is the quotient of a singly-periodic Scherk surface by a translation which has genus zero, four ends and total curvature $-4 \pi$, or

(iii) $M$ is the quotient of a doubly periodic Scherk surface by a commensurable lattice with genus zero, four ends, and total curvature $-4 \pi$, or

(iv) $M$ is the quotient of a helicoid by a translation which has genus zero, two ends and total curvature $-4 \pi$.

\section{The SPACES OF COMPACT INDEX ONE Minimal SURFACES}

Examples of compact index one minimal surfaces have been found by M. Ross [Ro1], who has proved that the classical Schwarz $\mathcal{P}$ and $\mathcal{D}$ minimal surfaces, as well as A. Schoen gyroid surface, have index one. He has also proved that close enough minimal surfaces to Schwarz $\mathcal{P}$ surface in flat three tori close enough to the cubic torus have also index one. Examples of genus two index one minimal surfaces in flat three space forms were obtained by Frohman and Hass [FH] by using Pitts minimax method.

Up to now, nothing is known about the existence of genus four index one minimal surfaces in flat three space forms. Recall that such surfaces must lie in flat three tori by Proposition 1.4. We should remark that there exist genus four Riemann surfaces on which there is only one meromorphic map of degree four, up to Möbius transformations ([FK, Theorem III.8.7]). Hence the algebraic geometric methods used in this paper cannot be applied to this situation. A quotient of A. Schoen I-WP-surface, see a description in [Kar2], gives an example of an embedded minimal surface whose conformal structure verifies this property. 
In this section we use the results in the previous section, in particular the total curvature bound given in Corollary 2.2, to study the spaces of compact index one minimal surfaces. Our main result is as follows:

Theorem 3.1. Let $\left\{M_{n}\right\}_{n \in \mathbb{N}}$ be a sequence of minimal surfaces in $\mathbb{R}^{3}$ with genus greater than two which are invariant by subgroups $G_{n}$ of isometries such that $\mathbb{R}^{3} / G_{n}$ is an orientable manifold with injectivity radius inj $\mathbb{R}^{3} / G_{n}=1$. Suppose that $M_{n} / G_{n}$ is a compact orientable surface with index one embedded in $\mathbb{R}^{3} / G_{n}$. Then:

(i) We can extract a convergent subsequence of $G_{n}$ to a discrete subgroup $G$ of isometries which is of the same type as $G_{n}$ for $n$ large enough.

(ii) We can extract a subsequence, denoted in the same way, of $M_{n}$ converging in the $C^{k}$ topology, $k \geq 2$, to an embedded $G$-invariant minimal surface $M$. In particular, the surfaces $M_{n} / G_{n}$ and $M / G$ are diffeomorphic for $n$ large enough and $M_{n} / G_{n} \rightarrow M / G$.

Proof. We sketch the main ideas of the proof and refer the reader to [RR2] for details.

First we establish uniform curvature estimates for the sequence of properly embedded surfaces $\left\{M_{n}\right\}_{n \in \mathbb{N}}$. Indeed, if we had not such estimates, a subsequence (denoted in the same way) of a rescaling of the surfaces would converge in the $C^{k}$ topology, for $k \geq 2$, to a catenoid in $\mathbb{R}^{3}$ since inj $\mathbb{R}^{3} / G_{n}=1$ and the index one condition is preserved when passing to the limit.

The arguments in [RR2, Assertion 3.1] prove that the total curvature of $M_{n} / G_{n}$ is the same as that of the catenoid for $n$ large enough. But this gives us a contradiction since by the Gauss-Bonnet Theorem the total curvature of $M_{n} / G_{n}$ equals $4 \pi(1-g)$, which is less than or equal to $-8 \pi$ as $g \geq 2$.

It is proved in [RR2, Theorem 2.5] that the uniform curvature estimate implies a local area bound for the sequence $\left\{M_{n}\right\}_{n \in \mathbb{N}}$. Hence, [RR2, Theorem 2.8], we can extract a convergent subsequence of $\left\{G_{n}\right\}_{n \in \mathbb{N}}$ to a discrete subgroup $G$ acting properly and discontinuously in $\mathbb{R}^{3}$, and a convergent subsequence in the $C^{k}$ topology, $k \geq 2$, of $\left\{M_{n}\right\}_{n \in \mathbb{N}}$ to a properly embedded minimal surface in $\mathbb{R}^{3}$ with multiplicity one. The surface $M$ is $G$-invariant.

Reasoning as in assertion 3.1 in [RR2], we conclude that the total curvature of $M / G$ is the same as that of $M_{n} / G_{n}$ for $n$ large enough. This shows that $M / G$ is non totally geodesic and hence it must have index one. We also deduce that $M / G$ must be compact since otherwise the total curvature of $M / G$ would be greater than $-8 \pi$ by Corollary 2.2 and this gives a contradiction as the total curvature of $M_{n} / G_{n}$ is less than or equal to $-8 \pi$.

As $M / G$ is compact [RR2, Theorem 2.8] shows that $M_{n} / G_{n}$ and $M / G$ are diffeomorphic for $n$ large and that $\mathbb{R}^{3} / G_{n}$ and $\mathbb{R}^{3} / G$ are affinely diffeomorphic for $n$ large enough. 
Remark. Frohman and Hass [FH, pp. 538-539] have constructed a family of genus two index one minimal surfaces by using Pitts minimax method in a flat three manifold which is covered three times by a flat three torus. Since the construction can be applied to a noncompact family of ambient manifold it yields a noncompact family of index one minimal surfaces.

We also have the following restriction on the ambient manifolds containing certain types of index one minimal surfaces.

Proposition 3.2. Let $M$ be a compact orientable index one minimal surface properly embedded in a complete orientable flat three manifold $N$.

If the genus of $M$ is four or $M$ is a genus three hyperelliptic surface then $N$ is a flat three torus.

Proof. Let $g=$ genus $M$. Under our hypotheses we can find on $M$ a holomorphic map $\phi$ of degree $g-1$ and so $|C(M)|=4 \pi(g-1)=4 \pi \operatorname{deg} \phi$. We conclude by applying Proposition 1.5.

Remark. A genus three minimal surface immersed in a flat three torus is hyperelliptic since its Gauss map has degree two.

\section{Applications to volume Preserving stable surfaces}

Recall that a constant mean curvature immersion $\psi: M \rightarrow N$ from a surface into a three manifold is volume preserving stable (v.p. stable) if the second derivative of the surface area is non-negative for any compactly supported variation keeping constant the volume enclosed by the surface. Analytically this condition is equivalent (for orientable two-sided surfaces) to

$$
\int_{M}\left\{|\nabla u|^{2}-\left(\operatorname{Ric}(\nu)+|\sigma|^{2}\right) u^{2}\right\} d A \geq 0,
$$

for all $u \in C^{\infty}(M)$ with compact support such that $\int_{M} u d A=0$ ([BCE]).

A minimal surface which is v.p. stable has index zero or one. If it compact, has index zero and the Ricci curvature of the ambient manifold is non-negative, then it is totally geodesic and so it is a torus. We conclude that an orientable two-sided compact minimal surface in a three-manifold with non-negative Ricci curvature which is v.p. stable and it is not a torus has index one.

Now we prove the following result

Theorem 4.1. Let $\psi: M \rightarrow N$ be a v.p. stable immersion from a compact orientable surface into an orientable three manifold with non-negative Ricci curvature.

Then the genus of $M$ is less than or equal to four. If the genus equals four then $M$ is an index one minimal surface whose Jacobi operator is the one associated to a holomorphic map of degree three. 
Proof. Under our hypotheses it is proved in [RR1, Theorem 1] that genus $M \leq 5$. Suppose that genus $M=4,5$. We shall prove that $M$ is an index one minimal surface whose Jacobi operator is the one associated to a holomorphic map of degree $g-1$. This implies the result by using (iii) in Proposition 1.2.

Consider a holomorphic map $\phi: M \rightarrow \mathbb{S}^{2}$ with degree less than or equal to $g-1$. This map can always be constructed since $g-1=1+[(g+1) / 2]$ for $g=4$, 5 , and can be taken with mean zero ([LY]). Using $\phi$ as a test function in (5) we obtain

$$
\begin{aligned}
8 \pi(g-1) & \geq \int_{M}\left(\operatorname{Ric}(\nu)+|\sigma|^{2}\right) \\
& =\int_{M} \operatorname{Ric}\left(e_{1}\right)+\operatorname{Ric}\left(e_{2}\right)+4 H^{2}-2 K \geq \int_{M}-2 K=8 \pi(g-1),
\end{aligned}
$$

where $H$ is the mean curvature of the immersion and $\left\{e_{1}, e_{2}\right\}$ is an orthonormal basis on $M$. We have used that the Ricci curvature of $N$ is non-negative in the last inequality and the Gauss-Bonnet Theorem in the last equality. As all the inequalities above become equalities we have $H=0, \operatorname{Ric}\left(e_{1}\right)=\operatorname{Ric}\left(e_{2}\right)=0$ and $\operatorname{deg} \phi=g-1$. Hence $M$ is a minimal surface whose index equals one since its genus is greater than one. Reasoning as in the proof of Proposition 1.1 we deduce that $|\nabla \phi|^{2}=-2 K=\operatorname{Ric}(\nu)+|\sigma|^{2}$ and so the Jacobi operator is given by $\Delta+|\nabla \phi|^{2}$, as we claimed.

\section{REFERENCES}

[BCE] J.L. Barbosa, M. do Carmo, J. Eschenburg, Stability of hypersurfaces with constant mean curvature in Riemannian manifolds, Math. Z., 197 (1988) 123-138.

[CP] M. do CARmo, C.K. Peng, Stable complete minimal surfaces in $\mathbb{R}^{3}$ are planes, Bull. Amer. Math. Soc. (N.S.), 1 (1979) 903-906.

[FK] H.M. FARKAS, I. KRA, Riemann surfaces, Lecture Notes on Mathematics, 71, Springer-Verlag, 1980.

[FC] D. Fischer-Colbrie, On complete minimal surfaces with finite Morse index in three manifolds, Invent. Math., 82 (1985) 121-132.

[FCS] D. Fischer-Colbrie, R. Schoen, The structure of complete stable minimal surfaces in 3-manifolds of non-negative scalar curvature, Comm. Pure Appl. Math., 33 (1980) 199-211.

[FH] C. Frohman AND J. HAss, Unstable minimal surfaces and Heegaard splittings, Invent. Math., 95 (1989) 529-540.

[GH] P. Griffiths, J. Harris, Principles of Algebraic Geometry, Pure and Applied Math., Wiley-Interscience series, 1978.

[HPR] J. Hass, J.T. PitTs, J.H. Rubinstein, Existence of unstable minimal surfaces in manifolds with homology and applications to triply periodic minimal surfaces, Proceedings of Symposia in Pure Mathematics, 1992.

[HM1] D. Hoffman, W.H. Meeks, Limits of minimal surfaces and Scherk's fifth surface, Arch. Rational Mech. Anal., 111 (1990), no. 2, 181-195.

[HM2] D. Hoffman, W.H. MeEks, The strong halfspace theorem for minimal surfaces, Invent. Math., 101 (1990) 373-377. 
[Kar1] H. KARChER, Embedded minimal surfaces derived from Scherk's examples, Manuscripta Math., 62 (1988) 83-114.

[Kar2] H. KARCHER, The triply periodic minimal surfaces of Alan Schoen and their constant mean curvature companions, Manuscripta Math., 64 (1989) 291-357.

[LY] P. Li AND S.T. YAU, A new conformal invariant and its applications to the Willmore conjecture and the first eigenvalue of compact surfaces, Invent. Math., 69 (1982) 269-291.

[LR] F.J. López, A. Ros, Complete minimal surfaces with index one and stable constant mean curvature surfaces, Comment. Math. Helv., 64 (1989) 34-43.

[MR1] W.H. Meeks, H. Rosenberg, The global theory of doubly periodic minimal surfaces, Invent. Math., 97 (1989) 351-379.

[MR2] W.H. Meeks, H. RosenberG, The geometry of periodic minimal surfaces, Comment. Math. Helv., 68 (1993) 538-578.

[MoR] S. Montiel, A. Ros, Schrödinger operators associated to a holomorphic map, Proceedings Conference on Global Differential Geometry and Global Analysis, Berlin, 1990, Lecture Notes on Mathematics 1481, 147-174.

[P] J.T. PITTs, Existence and regularity of minimal surfaces on Riemannian manifolds, Mathematical Notes, 27, Princeton University Press, Princeton, 1981.

[PR] J.T. PitTs, J.H. Rubinstein, Equivariant minimax and minimal surfaces in geometric three-manifolds, Bull. Amer. Math. Soc.(N.S.), 19 (1988), 303-309.

[RR1] M. RitorÉ, A. Ros, Stable constant mean curvature tori and the isoperimetric problem in three space-forms, Comment. Math. Helvetici, 67 (1992) 293-305.

[RR2] M. Ritoré, A. Ros, The spaces of index one minimal surfaces and stable constant mean curvature surfaces embedded in flat three manifolds, Transactions Amer. Math. Soc., 348, no. 1 (1996) 391-410.

[Ro1] M. Ross, Schwarz's $\mathcal{P}$ and $\mathcal{D}$ surfaces are stable, Differential Geom. Appl., 2 (1992) 179-195.

[Ro2] M. Ross, Complete nonorientable minimal surfaces in $\mathbb{R}^{3}$, Comment. Math. Helvetici, 67 (1992) 64-76.

[So] R. Sounm, Exemples d'applications holomorphes d'indice un, Ann. Inst. Fourier, 43, no. 2 (1993), 369-381.

[S] M. SoRET, Deformations des surfaces minimales, Thésé de Doctorat, Université Paris VII, 1993.

[Wo] J.A. Wolf, Spaces of constant curvature, 1st ed., Publish or Perish, Inc., 1984.

[Y] S.T. YAU, Nonlinear analysis in geometry, L'enseignement Math., 33 (1987), 109158.

This research was partially supported by DGICYT grant PB94-0731.

Departamento de Geometría y Topología

Universidad de Granada

E-18071 Granada, SPAIN

E-MAIL: ritore@ugr.es

Received: July 8th, 1996; revised: August 28th, 1997. 